Western University

Scholarship@Western

Physics and Astronomy Publications

Physics and Astronomy Department

$11-2-2011$

\title{
The Polylogarithm and the Lambert W Functions in Thermoelectrics
}

\author{
Muralikrishna Molli \\ Sri Sathya Sai Institute of Higher Learning, Prasanthinilayam, Andhra Pradesh, India, muralim.phy@gmail.com \\ Kamisetty Venkataramaniah \\ Sri Sathya Sai Institute of Higher Learning, Prasanthinilayam, Andhra Pradesh, India, vrkamisetti@gmail.com \\ Sree Ram Valluri \\ The University of Western Ontario, valluri@uwo.ca
}

Follow this and additional works at: https://ir.lib.uwo.ca/physicspub

Part of the Astrophysics and Astronomy Commons, and the Physics Commons

\section{Citation of this paper:}

Molli, Muralikrishna; Venkataramaniah, Kamisetty; and Valluri, Sree Ram, "The Polylogarithm and the Lambert W Functions in Thermoelectrics" (2011). Physics and Astronomy Publications. 23.

https://ir.lib.uwo.ca/physicspub/23 


\section{The Polylogarithm and the Lambert $W$ functions in Thermoelectrics}

Muralikrishna Molli, K. Venkataramaniah, S. R. Valluri

Muralikrishna Molli Department of Physics, Sri Sathya Sai Institute of Higher Learning, Prasanthinilayam, Andhra Pradesh, 515134, India.

K. Venkataramaniah Department of Physics, Sri Sathya Sai Institute of Higher Learning, Prasanthinilayam, Andhra Pradesh, 515134, India.

${ }^{1}$ S. R. Valluri Departments of Physics and Astronomy; Applied Mathematics, University of Western Ontario (UWO), Department of Business, Economics and Mathematics, Kings University College, UWO, Ontario N6A 5B7, Canada.

${ }^{1}$ Corresponding author e-mail: valluri@uwo.ca

Tel: (519) 661-2111 ext. 86499

Fax: (519) 661-3523

PACS numbers: Quantum statistical mechanics, 05.30.-d

Thermoelectric effects in semiconductors and insulators, 72.20.Pa

Special functions, 02.30.Gp

Accepted Oct. 18, 2011 by the Canadian Journal of Physics 


\begin{abstract}
In this work, we determine the conditions for the extremum of the figure of merit $\theta^{2}$, in a degenerate semiconductor for thermoelectric (TE) applications. We study the variation of the function $\theta^{2}$ with respect to the reduced chemical potential $\mu^{*}$ using relations involving polylogarithms of both integral and non-integral orders. We present the relevant equations for the thermopower, thermal, and electrical conductivities that result in optimizing $\theta^{2}$ and obtaining the extremum equations. We discuss the different cases that arise for various values of $r$, which depends on the type of carrier scattering mechanism present in the semiconductor. We also present the important extremum conditions for $\theta^{2}$ obtained by extremizing the TE power factor and the thermal conductivity separately. In this case, simple functional equations, which lead to solutions in terms of the Lambert W function, result. We also present some solutions for the zeros of the polylogarithms. Our analysis allows for the possibility of considering the reduced chemical potential and the index $r$ of the polylogarithm as complex variables.

Key words: Polylogarithms, Lambert W function, Thermoelectrics, Chemical Potential, Figure of Merit
\end{abstract}




\section{INTRODUCTION}

There is considerable current interest in quasizero-dimension physics that refers to the study of electrons and excitons in nanostructures. A polylogarithmic formulation of the statistical mechanics of ideal particles in null dimension could provide some useful insight and limits into and limits on the behavior of quantum dots. In recent years new methods [1-4] have been developed for the study of D-dimensional Bose and Fermi gases that have led to fruitful applications of the theory of Polylogarithms (hereinafter referred to as polylogs), also known as Jonquière's function [5] and closely related to the Lerch transcendent [6,7].

Polylogs are transcendental functions of two complex numbers. They are a modern generalization of Euler's dilogs and Landen's trilogs [8]. Polylogs have been studied by some of the greatest mathematicians of the past including Euler, Lambert, Legendre, Wirtinger, Abel, Appell, Lindelöf, Lobachevsky, Kummer, and Ramanujan among others. The dilogarithm function [9], also referred to as the Spence function, has also been connected with some of these great names in the history of mathematics and has successfully been used in quantum field theory by ' $t$ Hooft and Veltman [10] in connection with the Feynman diagram integrals for quantum processes, graviton calculations, algebraic geometry [11-13], and other applications. Polylogs have seen applications in fields such as combinatorics, knot theory, quantum statistics and high energy physics. These special functions are particularly useful in the study of the thermodynamics of Bose and Fermi statistics. Lee discussed the direct relationship between Fermi-Dirac (FD) integrals and polylogs in his pioneering work on the unification of Bose and Fermi statistics [14-16]. Different statistical effects refer to different structural properties of polylogs. One can view different statistics as representing different domains of polylogs.

Thermoelectric (TE) materials are those that are able to efficiently generate power using the Seebeck effect or refrigerate using the Peltier effect. TE materials are capable of acting as solid state refrigerators or heat pumps that do not use any moving parts or environmentally harmful fluids. Because of their high reliability and simplicity, TE materials are used extensively in fields such as space power generation, waste heat recovery, and a variety of cooling applications [17]. The potential of a material for TE applications is determined by a measure of the dimensionless 
parameter $\theta^{2}$ called the figure of merit. This parameter depends on the thermopower or Seebeck coefficient $\eta$, the electrical conductivity $\sigma$, the average temperature $T$ of the thermocouple, and the total thermal conductivity $\lambda$. The figure of merit plays a major role in the TE materials research, as the efficiency or the coefficient of performance of a TE couple is proportional to the figure of merit. To be competitive compared with conventional refrigerators and generators, one must have materials with figure of merit greater than three.

It is of theoretical, mathematical and experimental interest to determine the maximum figure of merit that can be achieved in TE materials.

This work focuses on the applications of the polylog and the Lambert $\mathrm{W}$ functions in thermoelectrics. In this work we determine the conditions for the extremum of the figure of merit in a degenerate semiconductor for TE applications. We study the variation of the function $\theta^{2}$ with respect to the reduced chemical potential using polylogs of both integral and non-integral order. In the special cases where the extrema of thermal conductivity and TE power factor are separately considered, simple functional equations that lead to solutions in terms of the Lambert $\mathrm{W}$ functions [18-21] result. The advantage of using polylogs and the Lambert W function is that they are built into standard computer mathematical packages such as Mathematica, Matlab, and Maple, eliminating the need for approximations or tabulations for Fermi-Dirac integrals. In section 2, we present the relevant equations for the thermopower, thermal, and electrical conductivity, that result in an optimized figure of merit $\theta^{2}$ and obtain the extremum equations for

$\theta^{2}$. In section 3, we discuss the different cases that arise for various values of $r$. In section 4, we also present the important extremum conditions for $\theta^{2}$ obtained by extremizing the TE power factor and the thermal conductivity separately as well as solutions for the zeros of the polylogs. The final section presents conclusions.

\section{The Extremum Conditions for $\theta^{2}$}

For a material to be a good TE material, it must have a high electrical conductivity, large Seebeck coefficient, and low thermal conductivity. Insulators are poor TE materials owing to their low electrical conductivity. Despite having high electrical conductivity, metals are not 
favorable for TE applications because of their extremely low Seebeck coefficient values [22]. On the other hand, Seebeck coefficient values of semiconductors are much higher than those of metals [23]. Several semiconductors have a high melting point which permits their operation at high temperatures. The power factor, $\eta^{2} \sigma$, can be optimized in semiconductors through doping to give the largest figure of merit[24]. The ratio of the thermal to the electrical conductivity, $\lambda / \sigma$ , could be decreased if the semiconductor TE material is alloyed with an isomorphous element or compound[25]. These factors make semiconductors a good choice for TE applications.

We consider a thermocouple whose branches are made up of $n$-and $p$-type semiconductors of the same material and of the same electrical conductivity. The relevant equations for $\theta^{2}$ and its extremum are presented later in detail. Some of them are taken from the literature [22]. The figure of merit is given by

$$
\theta^{2}=\frac{\eta^{2} \sigma T}{\lambda}
$$

The thermal conductivity, $\lambda$, contains two components: the electronic component, $\lambda_{e}$, and a lattice component, $\lambda_{0}$ :

$$
\lambda=\lambda_{e}+\lambda_{0}
$$

$\eta^{2} \sigma$ is called the TE power factor.

The coefficient of performance (refrigeration mode) [22] and the efficiency (power generation mode) [24] of the TE couple are proportional to the figure of merit.

The expressions for $\eta, \sigma$ and $\lambda_{e}$ for an extrinsic semiconductor are given by $[22,24]$

$$
\begin{aligned}
& \eta=\frac{k}{e}\left[\frac{(r+2) F_{r+1}}{(r+1) F_{r}}-\mu^{*}\right] \\
& \sigma=\frac{16 \pi m e^{2} l_{0}(k T)^{r+1}(r+1) F_{r}}{3 h^{3}} \\
& \lambda_{e}=\frac{16 \pi m l_{0} k^{r+3} T^{r+2}}{3 h^{3}}\left[(r+3) F_{r+2}-\frac{(r+2)^{2} F_{r+1}^{2}}{(r+1) F_{r}}\right]
\end{aligned}
$$

where $k$ is the Boltzmann constant, $e$ is the electronic charge, $\mu^{*}$ is the reduced chemical potential equal to the Fermi level divided by $k T, m$ is the effective mass of the carriers, $l_{0}$ is a 
proportionality constant in the expression for the carrier mean free path $\left(l=l_{0} E^{r}\right), E$ is the carrier energy, and $h$ is the Planck's constant.

The functions $F_{i}(i=r, r+1, r+2)$ are Fermi integrals of the form

$$
F_{i}\left(\mu^{*}\right)=\int_{0}^{\infty} \frac{x^{i} d x}{e^{x-\mu^{*}}+1} .
$$

Physicists first became aware of the FD integrals through the work of Sommerfeld [26].

McDougall and Stoner, in addition to tabulating them, explored a number of analytic aspects of such functions [27].

The quantity $r$ takes on different values depending on the carrier scattering mechanism:

for lattice scattering in a non-polar material and a polar material, $r=0$ and $1 / 2$, respectively; for ionized impurity scattering $r=2$ [22].

When the chemical potential becomes complex, the FD integral can be generalized to make the index $r$ of the polylog functions a complex variable. Such a generalization would lead to a richer structure of solutions for the zeros of the polylog functions. The zeros will have a possible close connection to the zeros of the Riemann $\zeta$ function [28].

We derive conditions that give the extremum of the figure of merit with respect to the reduced chemical potential.

At a given operating temperature $T$, in order to obtain the extremum of $\theta^{2}$, we equate the derivative of $\left(\eta^{2} \sigma / \lambda\right)$ with respect to $\mu^{*}$ with zero; that is,

$$
\frac{d}{d \mu^{*}}\left(\frac{\eta^{2} \sigma}{\lambda}\right)=0
$$

which leads to the following cases:

$$
\begin{array}{r}
\left(\frac{\eta^{2} \sigma}{\lambda}\right)=0 \\
\text { or } \\
{\left[\frac{1}{\sigma} \frac{d \sigma}{d \mu^{*}}+\frac{2}{\eta} \frac{d \eta}{d \mu^{*}}-\frac{1}{\lambda} \frac{d \lambda}{d \mu^{*}}\right]=0}
\end{array}
$$


Because $\lambda_{0}$ is independent of $\mu^{*}$ in (8), we have $\frac{d \lambda}{d \mu^{*}}=\frac{d}{d \mu^{*}}\left(\lambda_{e}+\lambda_{0}\right)=\frac{d \lambda_{e}}{d \mu^{*}}$.

To evaluate the various derivatives, we rewrite the expressions for $\sigma, \eta$ and $\lambda_{e}$ in terms of polylogs, as the Fermi integrals are a subset of the polylogs [14, 29]:

$$
F_{r}\left(\mu^{*}\right)=-\Gamma(r+1) L i_{r+1}\left(-\exp \mu^{*}\right)
$$

From (3), (4), (5) and (9) we obtain the following expressions:

$$
\begin{gathered}
\eta=\frac{k}{e}\left[(r+2) \frac{L i_{r+2}\left(-\exp \mu^{*}\right)}{L i_{r+1}\left(-\exp \mu^{*}\right)}-\mu^{*}\right] \\
\sigma=\frac{-16 \pi m e^{2} l_{0}(k T)^{r+1} \Gamma(r+2)}{3 h^{3}} L i_{r+1}\left(-\exp \mu^{*}\right) \\
\lambda_{e}=\frac{16 \pi m l_{0} k(k T)^{r+2}}{3 h^{3}}\left[-\Gamma(r+4) L i_{r+3}\left(-\exp \mu^{*}\right)+(r+2) \Gamma(r+3) \frac{\left[L i_{r+2}\left(-\exp \mu^{*}\right)\right]^{2}}{L i_{r+1}\left(-\exp \mu^{*}\right)}\right]
\end{gathered}
$$

In obtaining the preceding equations, we used the recurrence formula for the gamma function

$$
r \Gamma(r)=\Gamma(r+1)
$$

For the sake of brevity, hereinafter we write mostly $L i_{r}$ for $L i_{r}\left(-\exp \mu^{*}\right)$.

From (10), (11) and (12), we obtain the following derivatives:

$$
\begin{gathered}
\frac{d \eta}{d \mu^{*}}=\frac{k}{e}\left\{(r+2)\left[1-\frac{L i_{r} L i_{r+2}}{L i_{r+1}^{2}}\right]-1\right\} \\
\frac{d \sigma}{d \mu^{*}}=\frac{-16 \pi m e^{2} l_{0}(k T)^{r+1} \Gamma(r+2)}{3 h^{3}} L i_{r} \\
\frac{d \lambda_{e}}{d \mu^{*}}=\frac{16 \pi m l_{0} k(k T)^{r+2}}{3 h^{3}}\left\{-\Gamma(r+4) L i_{r+2}-(r+2) \Gamma(r+3)\left[\frac{L i_{r+2}{ }^{2} L i_{r}}{L i_{r+1}{ }^{2}}-2 L i_{r+2}\right]\right\}
\end{gathered}
$$

In obtaining the preceding derivatives, we used the recurrence relation for polylogs [30]:

$$
z \frac{d}{d z} L i_{s+1}(z)=L i_{s}(z) .
$$

Substituting equations (10) -(15) into (8), we get the following condition for the extremum of $\theta^{2}$ : 


$$
\frac{2\left\{(r+2)\left[L i_{r+1}^{2}-L i_{r} L i_{r+2}\right]-L i_{r+1}^{2}\right\}}{(r+2) L i_{r+2} L i_{r+1}-\mu^{2} L i_{r+1}^{2}}+\frac{L i_{r}}{L i_{r+1}}-\frac{D\left\{-\Gamma(r+4) L i_{r+2}-(r+2) \Gamma(r+3)\left[\frac{L i_{r+2}^{2} L i_{r}}{L i_{r+1}^{2}}-2 L i_{r+2}\right]\right\}}{\lambda_{0}+D\left\{(r+2) \Gamma(r+3) \frac{L i_{r+2}{ }^{2}}{L i_{r+1}}-\Gamma(r+4) L i_{r+3}\right\}}=0
$$

where

$D=\left(16 \pi m l_{0} k(k T)^{r+2} / 3 h^{3}\right)$.

Equation (16) gives the extremum equation for the general case of $r$. Now we shall obtain the simplified extremum equations for specific values of $r(0,1 / 2$ and 2$)$.

\section{Three Different Cases for $r$}

\section{Case 1}

$r=0$

Substituting $r=0$ into equation (16) and simplifying we get the following:

$$
\begin{gathered}
L i_{1}^{3}\left\{\left(\lambda_{0}-6 D L i_{3}\right)\left(2 L i_{1}^{2}-2 L i_{0} L i_{2}-\mu^{*} L i_{0} L i_{1}\right)+2 D L i_{2}\left(\mu^{*} L i_{1}^{2}-4 \mu^{*} L i_{0} L i_{2}+2 L i_{1} L i_{2}\right)\right\}=0 \\
\Rightarrow L i_{1}=0 \\
\text { or } \\
\left\{\left(\lambda_{0}-6 D L i_{3}\right)\left(2 L i_{1}^{2}-2 L i_{0} L i_{2}-\mu^{*} L i_{0} L i_{1}\right)+2 D L i_{2}\left(\mu^{*} L i_{1}^{2}-4 \mu^{*} L i_{0} L i_{2}+2 L i_{1} L i_{2}\right)\right\}=0
\end{gathered}
$$

This gives various possibilities as discussed in the following list.

(i) If

$$
\left(\lambda_{0}-6 D L i_{3}\right)=0
$$

then,

$$
\begin{gathered}
2 D L i_{2}\left(\mu^{*} L i_{1}^{2}-4 \mu^{*} L i_{0} L i_{2}+2 L i_{1} L i_{2}\right)=0 \\
\Rightarrow L i_{2}=0 \\
\text { or } \\
\left(\mu^{*} L i_{1}^{2}-4 \mu^{*} L i_{0} L i_{2}+2 L i_{1} L i_{2}\right)=0(\because D \neq 0)
\end{gathered}
$$

In this case, which started from the analysis of (19), the following are the conditions for the extremum of $\theta^{2}$ in addition to (18): 


$$
\begin{array}{r}
L i_{2}=0 \\
L i_{3}=\frac{\lambda_{0}}{6 D} \\
\left(\mu^{*} L i_{1}^{2}-4 \mu^{*} L i_{0} L i_{2}+2 L i_{1} L i_{2}\right)=0
\end{array}
$$

(ii) From (19), if we consider one of the other possibilities

$$
\left(2 L i_{1}^{2}-2 L i_{0} L i_{2}-\mu^{*} L i_{0} L i_{1}\right)=0
$$

then (21) holds, and (22) or (23).

In this case, which started from the analysis of (19), the following are the conditions for the extremum of $\theta^{2}$ in addition to (18) - (24), (26), and (27).

(iii) From (19), if we consider the third possibility, (24), then

$$
\left(\lambda_{0}-6 D L i_{3}\right)\left(2 L i_{1}^{2}-2 L i_{0} L i_{2}-\mu^{*} L i_{0} L i_{1}\right)=0
$$

which leads to (25) or (27).

In this case of (iii) which started from an analysis of (19), the following are the conditions for the extremum of $\theta^{2}$ in addition to (18): (24), (25), and (27):

(iv) From (19), if one considers the fourth possibility, (26), one finds that (28) leads to (25) or (27).

In this case, which started from the analysis of (19), the following are the conditions for the extremum of $\theta^{2}$ in addition to (18): (25), (26), and (27).

\section{Case 2}

$r=1 / 2$

Substituting $r=1 / 2$ into equation (16) and simplifying, one gets the following:

$$
\begin{gathered}
L i_{\frac{3}{2}}^{3}\left[\left(2 \lambda_{0}-7 D \Gamma\left(\frac{7}{2}\right) L i_{\frac{7}{2}}\right)\left(6 L i_{\frac{3}{2}}{ }^{2}-5 L i_{\frac{1}{2}} L i_{\frac{5}{2}}-2 \mu^{*} L i_{\frac{1}{2}} L i_{\frac{3}{2}}\right)+D \Gamma\left(\frac{7}{2}\right) L i_{\frac{5}{2}}\left(6 \mu^{*} L i_{\frac{3}{2}}{ }^{2}-20 \mu^{*} L i_{\frac{1}{2}} L i_{\frac{5}{2}}+15 L i_{\frac{3}{2}} L i_{\frac{5}{2}}\right)\right]=0 \\
\Rightarrow L i_{\frac{3}{2}}=0
\end{gathered}
$$

or

$$
\left[\left(2 \lambda_{0}-7 D \Gamma\left(\frac{7}{2}\right) L i_{\frac{7}{2}}\right)\left(6 L i_{\frac{3}{2}}{ }^{2}-5 L i_{\frac{1}{2}} L i_{\frac{5}{2}}-2 \mu^{*} L i_{\frac{1}{2}} L i_{\frac{3}{2}}\right)+D \Gamma\left(\frac{7}{2}\right) L i_{\frac{5}{2}}\left(6 \mu^{*} L i_{\frac{3}{2}}{ }^{2}-20 \mu^{*} L i_{\frac{1}{2}} L i_{\frac{5}{2}}+15 L i_{\frac{3}{2}} \frac{L}{\frac{5}{2}}\right)\right]=0
$$


This gives various possibilities as discussed later.

(v) If

$$
\left(2 \lambda_{0}-7 D \Gamma\left(\frac{7}{2}\right) L i_{\frac{7}{2}}\right)=0
$$

the following are the conditions for the extremum of $\theta^{2}$ apart from (30):

$$
\begin{gathered}
L i_{\frac{5}{2}}=0 \\
L i_{\frac{7}{2}}=\frac{2 \lambda_{0}}{7 D \Gamma\left(\frac{7}{2}\right)} \\
\left(6 \mu^{*} L i_{\frac{3}{2}}^{2}-20 \mu^{*} L i_{\frac{1}{2}} L i_{\frac{5}{2}}+15 L i_{\frac{3}{2}} L i_{\frac{5}{2}}\right)=0
\end{gathered}
$$

(vi) If

$$
\left(6 L i_{\frac{3}{2}}{ }^{2}-5 L i_{\frac{1}{2}} L i_{\frac{5}{2}}-2 \mu^{*} L i_{\frac{1}{2}} L i_{\frac{3}{2}}\right)=0
$$

the following conditions result for the extremum of $\theta^{2}$ apart from (30): (33), (35), and (36).

(vii) If (33) is true,

the following conditions result for the extremum of $\theta^{2}$ apart from (30): (33), (34), and (36)

(viii) If (35) is true, the following conditions result for the extremum of $\theta^{2}$ apart from (30): (34), (35), and (36).

\section{Case 3}

We will consider $r=2$ next.

Substituting $r=2$ into (16) and simplifying we get the following:

$$
\begin{gathered}
L i_{3}^{3}\left\{\left(\lambda_{0}-120 D L i_{5}\right)\left(6 L i_{3}{ }^{2}-4 L i_{2} L i_{4}-\mu^{*} L i_{2} L i_{3}\right)+24 D L i_{4}\left(3 \mu^{*} L i_{3}{ }^{2}-8 \mu^{*} L i_{2} L i_{4}+12 L i_{3} L i_{4}\right)\right\}=0 \\
\Rightarrow L i_{3}=0
\end{gathered}
$$

or

$$
\left\{\left(\lambda_{0}-120 D L i_{5}\right)\left(6 L i_{3}^{2}-4 L i_{2} L i_{4}-\mu^{*} L i_{2} L i_{3}\right)+24 D L i_{4}\left(3 \mu^{*} L i_{3}^{2}-8 \mu^{*} L i_{2} L i_{4}+12 L i_{3} L i_{4}\right)\right\}=0
$$


This gives various possibilities as discussed in the following list.

(ix) If

$$
\left(\lambda_{0}-120 D L i_{5}\right)=0
$$

the following are the conditions for the extremum of $\theta^{2}$ in addition to (38):

$$
\begin{gathered}
L i_{4}=0 \\
L i_{5}=\frac{\lambda_{0}}{120 D} \\
\left(3 \mu^{*} L i_{3}{ }^{2}-8 \mu^{*} L i_{2} L i_{4}+12 L i_{3} L i_{4}\right)=0
\end{gathered}
$$

(x) If

$$
\left(6 L i_{3}^{2}-4 L i_{2} L i_{4}-\mu^{*} L i_{2} L i_{3}\right)=0
$$

the following conditions result for the extremum of $\theta^{2}$ in addition to (39): (41), (43), and (44).

(xi) If (41) is true, then the following conditions result for the extremum of $\theta^{2}$ in addition to (38): (41), (42), and (44).

(xii) If (43) is true, then the following conditions result for the extremum of $\theta^{2}$ in addition to (39): (42), (43), and (44).

In the following section, we consider special and also equally important cases where the TE power factor and the thermal conductivity are extremized separately. This can also be of great importance for experimentalists, who may be confronted with the problem of deciding which of these would be easier to study or implement [31].

\section{Extremization of the TE power factor and thermal conductivity separately}

The extremization of the TE power factor $\left(\eta^{2} \sigma\right)$ leads to the following equations:

$$
\left(\eta^{2} \sigma\right)=0
$$




$$
\left[\frac{1}{\sigma} \frac{d \sigma}{d \mu^{*}}+\frac{2}{\eta} \frac{d \eta}{d \mu^{*}}\right]=0
$$

which, on simplification, gives the following equation:

$$
\frac{2\left\{(r+2)\left[L i_{r+1}^{2}-L i_{r} L i_{r+2}\right]-L i_{r+1}^{2}\right\}}{(r+2) L i_{r+2} L i_{r+1}-\mu^{*} L i_{r+1}^{2}}+\frac{L i_{r}}{L i_{r+1}}=0
$$

Further algebraic manipulation leads to the following result

$$
2(r+1) L i_{r+1}^{3}-(r+2) L i_{r} L i_{r+1} L i_{r+2}-\mu^{*} L i_{r} L i_{r+1}{ }^{2}=0
$$

The extremization of the thermal conductivity $(\lambda)$ leads to the following equations:

$$
D\left\{-\Gamma(r+4) L i_{r+2}-(r+2) \Gamma(r+3)\left[\frac{L i_{r+2}^{2} L i_{r}}{L i_{r+1}^{2}}-2 L i_{r+2}\right]\right\}=0
$$

where $D \neq 0$ when $T \neq 0$.

This can be simplified to

$$
[\Gamma(r+4)-2 \Gamma(r+3)] L i_{r+1}^{2} L i_{r+2}-(r+2) \Gamma(r+3) L i_{r} L i_{r+2}^{2}=0
$$

These equations are simplified for the three separate cases of $r(r=0,1 / 2$ and 2$)$ that follow.

\section{Case 1}

$r=0$

Equation (48) reduces to the following equations

$$
L i_{1}=0
$$

and

$$
2 L i_{1}^{2}-2 L i_{0} L i_{2}-\mu^{*} L i_{0} L i_{1}=0
$$

Equation (50) reduces to the following equations:

$$
L i_{2}=0
$$

and

$$
L i_{1}^{2}-2 L i_{0} L i_{2}=0
$$

It is observed that equations (51), (52) and (53), (54) hold simultaneously on extremizing $\theta^{2}$. Now (52) can be simplified further with the aid of (54) to give the following equations:

$$
L i_{1}=0
$$

and 


$$
L i_{1}=\mu^{*} L i_{0}
$$

To summarize, the conditions for the case $r=0$ are

$$
\begin{gathered}
L i_{1}\left(-\exp \mu^{*}\right)=0 \\
L i_{2}\left(-\exp \mu^{*}\right)=0 \\
L i_{1}\left(-\exp \mu^{*}\right)=\mu^{*} L i_{0}\left(-\exp \mu^{*}\right)
\end{gathered}
$$

\section{Case 2}

$r=1 / 2$

In a similar approach that was followed for $r=0$, one obtains the following equations:

$$
\begin{gathered}
L i_{\frac{3}{2}}\left(-\exp \mu^{*}\right)=0 \\
L i_{\frac{5}{2}}\left(-\exp \mu^{*}\right)=0 \\
L i_{\frac{3}{2}}\left(-\exp \mu^{*}\right)=\frac{2}{3} \mu^{*} L i_{\frac{1}{2}}\left(-\exp \mu^{*}\right)
\end{gathered}
$$

\section{Case 3}

$r=2$

The conditions given later for this case are obtained in a similar way as in the previous cases.

$$
\begin{gathered}
L i_{3}\left(-\exp \mu^{*}\right)=0 \\
L i_{4}\left(-\exp \mu^{*}\right)=0 \\
L i_{3}\left(-\exp \mu^{*}\right)=\frac{\mu^{*}}{3} L i_{2}\left(-\exp \mu^{*}\right)
\end{gathered}
$$

We find interesting solutions for the different cases that follow.

\section{Case 1}

$z=-\exp \mu^{*},|z|<<1,\left|\mu^{*}\right|<<1$ and $\mu^{*}<0$

It is interesting to observe that one gets the following solutions for $L i_{s}(z)=0$ where $s$ can take any one of three numbers $(0,1 / 2$ and 2$)$ or a more general value

$$
-\left(\mu^{*}+\frac{3^{s}+1}{2}\right)=W_{j}\left(-\frac{3^{s}}{2^{s+1}} e^{-\left(\frac{3^{s}+1}{2}\right)}\right)
$$




$$
\mu^{*}=-\left\{\frac{3^{s}+1}{2}+W_{j}\left(-\frac{3^{s}}{2^{s+1}} e^{-\left(\frac{3^{s}+1}{2}\right)}\right)\right\}
$$

where $W_{j}()$ is the multi-valued Lambert $W$ function for branch $j$.

It is of interest to observe that we used a trinomial equation in $z$ and an approach similar to Euler's in solving such an equation $[32,33]$.

For $s=3 / 2$, the values of $W_{j}(j=0,-1$ and 1$)$ and $\mu^{*}$ are shown in Table 1 .

Fornberg and Kölbig [28] did a detailed investigation of the complex zeros of the polylog function, for real values of $z$ and complex $r$, and found that there may be trajectories of the zeros that tend towards the zeros of the Riemann $\zeta$ function as $z \rightarrow-1$.

\section{Case 2}

$$
z=-\exp \mu^{*},|z|<<1,\left|\mu^{*}\right|>1 \text { and } \mu^{*}<0
$$

In this case, we get (69), given below, as a solution for $\operatorname{Li}_{s}(z)=0$.

The series expansion for $\operatorname{Li}_{s}(z)$ is given below for $|z|<1$

$$
L i_{s}(z)=\sum_{k=1}^{\infty} \frac{z^{k}}{k^{s}}
$$

They are defined in the complex plane over the open unit disk and can be uniquely extended over the whole complex plane by analytic continuation.

For sufficiently small $z,\left|\mu^{*}\right|>1$ and $\mu^{*}<0$, we considered the first three terms in the preceding series to approximately solve for the zeros of the polylogs. The analysis can be generalized to include a large number of terms in the series, say $k=N(N>>1)$, and retain only the first two terms of the exponential for each power of $z$.

$$
\mu^{*}=\frac{1}{2}\left\{s \ln 4-W_{j}\left(-\frac{2}{3^{s}} e^{s \ln 4}\right)\right\}
$$

\section{Case 3}

$z=-\exp \mu^{*},|z|>1$, and $\mu^{*}>0$

For integer $r(>1)$, we have the following identity 


$$
L i_{r}(z)+(-1)^{r} L i_{r}\left(\frac{1}{z}\right)=\frac{-2 \pi i}{r !} B_{r}\left(\frac{\log z}{2 \pi i}\right)
$$

Here $B_{r}(x)$ is a Bernoulli polynomial given by

$$
B_{r}(x)=x^{r}-\frac{1}{2} r x^{r-1}+\left(\begin{array}{l}
r \\
2
\end{array}\right) B_{1} x^{r-2}-\left(\begin{array}{l}
r \\
4
\end{array}\right) B_{2} x^{r-4}+\ldots
$$

and $B_{0}=1, B_{1}=-1 / 2, B_{2}=1 / 6, B_{3}=0, B_{4}=-1 / 30 \ldots$

If $L i_{r}(z)=0,(70)$ simplifies as

$$
(-1)^{r} L i_{r}\left(-\exp \left(-\mu^{*}\right)\right)=\frac{-2 \pi i}{r !} B_{r}\left(\frac{1}{2}-i \frac{\mu^{*}}{2 \pi}\right)
$$

For example, $r=2$ gives the following solution for $\mu^{*}<<1$ :

$$
-e^{-\mu^{*}}\left(a+b \mu^{*}\right)=i\left(\frac{3 \pi}{4}+\frac{\mu^{*}}{4 \pi}\right)
$$

where

$a=\left(\frac{1}{1^{2}}-\frac{1}{2^{2}}+\frac{1}{3^{2}}-\frac{1}{4^{2}}+\frac{1}{5^{2}}-\frac{1}{6^{2}}+\ldots\right)$

and

$b=\left(\frac{1}{2^{2}}-\frac{2}{3^{2}}+\frac{3}{4^{2}}-\frac{4}{5^{2}}+\frac{5}{6^{2}}-\frac{6}{7^{2}}+\ldots\right)$

Equation (73) further simplifies to the following:

$$
-c\left(\mu^{*}+d\right)=W_{j}\left(\frac{3 i \pi c}{4 b} e^{-c d}\right)
$$

where

$$
c=\left(1+\frac{1}{3 \pi^{2}}\right)
$$

and

$$
d=\frac{a}{b}
$$

\section{Case 4}

$z=-\exp \mu^{*}, \mu^{*}>>1$, and $|z|>>1$

For non-integral order $(r)$, we have the following inversion relation valid for $\log (-z)>>1$ : 


$$
(-1)^{-r+1} L i_{r+1}\left(\frac{1}{z}\right)+L i_{r+1}(z)=-\sum_{n=0,1,2 \ldots} \frac{A_{2 n} \alpha^{r+1-2 n}}{\Gamma(r+2-2 n)}
$$

where $\alpha=\log (-z)$, and $A_{2 n}=-2 L i_{2 n}(-1)$.

It is worth mentioning that the case of non-integral order is also of long-standing interest. In fact, for $r=-1 / 2,1 / 2$ and $3 / 2$, Truesdell [28, 34] has investigated the zeros of the polylogs in the theory of the structure of polymers in detail.

For example, $r=1 / 2$ gives the following solution :

$$
\begin{gathered}
i \operatorname{Li}_{\frac{3}{2}}\left(-\exp \left(-\mu^{*}\right)\right)=-\left[\frac{4}{3 \sqrt{\pi}}\left(\mu^{*}\right)^{3 / 2}+\frac{\pi^{3 / 2}}{6}\left(\mu^{*}\right)^{-1 / 2}\right] \\
\mu^{*}=\frac{3}{2} W_{j}\left\{\frac{2}{3}\left(\frac{3 \sqrt{\pi}}{4}\right)^{2 / 3} e^{i \pi / 3}\right\}
\end{gathered}
$$

Another interesting way of maximizing figure of merit is when the electronic contribution to thermal conductivity, $\lambda_{e}$, equals zero. Under this situation equation (12) leads to the following condition:

$$
(r+2) L i_{r+2}^{2}-(r+3) L i_{r+1} L i_{r+3}=0
$$

\section{Concluding remarks}

We have derived the conditions for the extremum of the figure of merit $\theta^{2}$ in a degenerate semiconductor for TE applications. In deriving these conditions, we used the expressions for the FD integrals in terms of polylogs. The extremum conditions obtained involve zeros of the polylogs as well as functional equations in polylogs. For the case where the TE power factor and the thermal conductivity are extremized separately, a subset of conditions involved in the general case result. For most of these conditions, it is feasible to derive solutions for the reduced chemical potential, $\mu^{*}$, in terms of the multi-branched Lambert $\mathrm{W}$ function, $W_{j}$. The reduced chemical potential $\mu^{*}$ is an important thermodynamic variable that describes both classical and quantum mechanical states of a system [35]. Our analysis is applicable to the case where $\mu^{*}$, the argument $z$, and the index $r$ of the polylog can be complex [36]. 
An important problem that arises in our work is the zeros of the polylogs of non-integral order. This will be of great potential interest in the current technological drive towards developing more efficient TE materials. The solutions obtained for $\mu^{*}$ provide values for the Fermi energy level that extremize the figure of merit. These will be a good guide for experimental investigation. The challenge of developing more efficient TE materials will warrant a thorough study of quantum and fractional statistics to complement experimental investigations. These applications strongly indicate the immense potential and utility of polylogs and the Lambert W functions not only in quantum statistics but to other diverse practical problems [37].

Mathematicians have made an intensive analytical and numerical study of polylogs. Vepštas [38] has extended the techniques given by Borwein for computing the Riemann $\zeta$ function and provided an algorithm for evaluation of the polylogs for complex $r$ in a limited domain for $z$. By using the duplication and inversion formulae, his algorithm allows for the evaluation of the polylogs for all complex $r$ and $z$. We plan to do a detailed numerical analysis of the zeros of polylogs that arise in our study of thermoelectrics with the aid of the preceding recent mathematical research done in this area. We also plan to test these results experimentally in nanostructured bismuth telluride as well as alkali metal doped bismuth telluride for low temperature cooling applications and in nanostructured vanadium pentoxide as well as alkali metal doped vanadium pentoxide for high temperature power generation applications.

The presence of logarithmic, FD or Bose-Einstein integrals, the Riemann $\zeta$ functions is a strong attractor to welcome polylogs for the analysis of problems. The FD as well as Bose-Einstein integrals of both integral and non-integral order that can be cast into polylogs facilitate fast and precise calculations using mathematical packages or numerical methods. In light of the remarkable applications of the classical polylog and the polypseudologarithms [39] in physical problems, it will not be unexpected for the more general multiple polylog to occur in a variety of physical contexts [40].

\section{Acknowledgements}

M. M. and K. V. acknowledge the Council of Scientific and Industrial Research (CSIR), Govt. of India, for a Research Fellowship. S. R. V. would like to thank Kings University College and the 
Faculty of Science, University of Western Ontario (UWO), for a research grant during this work. We would also like to thank H. Lee (University of Georgia, Athens), N. Temme (CWI, Amsterdam) and J. Drozd of (Robarts Research Institute, UWO) for their constructive critique on our work.

\section{References}

1. R. K. Pathria. Statistical Mechanics. Pergamon, Oxford. 1972.

2. W. A. Barker. J. Math. Phys. 27, 302 (1986); 1385 (1987); 28, 1389 (1987).

3. M. H. Lee. J. Math. Phys. 30, 1837 (1989).

4. M. D. Kostin. J. Math. Phys. 35, 2371 (1994); 36, 750 (1995).

5. A. Jonquière. Note sur la série $\sum_{n=1}^{\infty} \frac{x^{n}}{n^{s}}$. Öfversigt af Kongl. Vetenskaps- Akademiens

Förhandlingar 46, 257-268 (1889a).

6. M. Lerch. Note sur la function $\mathfrak{A}(w, x, s)=\sum_{k=0}^{\infty} \frac{e^{2 k \pi i x}}{(w+k)^{2}}$ Acta Math. 11, 19-24 (1887).

7. A. Erdèlyi, W. Magnus, F. Oberhettinger and F.G. Tricomi. Higher Transcendental

Functions, 1, McGraw-Hill, New York, 1953, reprinted Krieger, Melbourne, FL, 1981.

8. L. Lewin. Polylogarithms and Associated Functions. North Holland, New York. 1981.

9. L.C. Maximon. Proc. R. Soc. Lond. A. 459, 2807-2819 (2003).

10. G. 't Hooft and M. Veltman. Nucl. Phys. B. 153, 365 - 401 (1979).

11. D. Zagier. Progr. Math. 89, 391-430 (1991).

12. A.E. Dubinov and A. A. Dubinova. Plasma Phys. Rep. 34, 403 (2008).

13. Bruce C. Berndt. Ramanujan's Notebooks Part I (1985), Springer Verlag, New York. 14. M. H. Lee. J. Math. Phys. 36, 1217 (1995).

15. M. H. Lee. Phys. Rev. E. 53, 548 (1996).

16. M. H. Lee. Phys. Rev. E. 54, 946 (1996).

17. Terry M. Tritt. Science. 272, 1276 (1996).

18. J. Tanguay, M. Gil, D. J. Jeffrey and S.R. Valluri. J. Math. Phys. 51, 123303 (2010).

19. S.R. Valluri, M. Gil, D. J. Jeffrey and S. Basu. J. Math. Phys. 50, 102103 (2009).

20. R. M. Corless, G. H. Gonnet, D. E. G. Hare, D. J. Jeffrey, and D. E. Knuth. Adv. Comput. Maths. 5, 329 (1996).

21. S. R. Valluri, D. J. Jeffrey, and R. M. Corless. Can. J. Phys. 78, 823 (2000). 
22. E. S. Rittner. J. Appl. Phys. 30(5), $702-707$ (1959).

23. H. J. Goldsmid and R. W. Douglas. Br. J. Appl. Phys. 5, 386-390 (1954).

24. A. F. Ioffe. Semiconductor Thermoelements and Thermoelectric Cooling. Infosearch Limited, London. 1957.

25. A. F. Ioffe, S. V. Airapetyants, A. V. Ioffe, N. V. Kolomoets, L. S. Stilbans. Dokl. Akad. Nauk SSSR. 106, 931 (1956).

26. A. Sommerfeld. Z. Physik. 47, 1 (1928).

27. J. McDougall and E.C. Stoner. Phil. Trans. R. Soc. Lond. A. 237, 67 (1938).

28. B. Fornberg and K. S. Kölbig. Math. Comp. 29, 582-599 (1975).

29. Marc D. Ulrich, William F. Seng, and Peter A. Barnes. J. Comput. Electron. 1, 431 (2002).

30. M. H. Lee. Phys. Rev. E. 56, 3909 (1997).

31. T. M. Tritt and M. A. Subramanian. MRS Bulletin. 31(3), 188-194 (2006).

32. J.H. Lambert. "Observationes variae in mathesin puram" Acta Helvetica, physicomathematico-anatomico-botanico-medica, 3, Basel, (1758) 128-168; "Observations Analytiques" in Nouveaux mémoires de l'Académie royale des sciences et belles-lettres, Berlin, 1772, vol. 1, for 1770.

33. L. Euler. "De serie Lambertina plurimisque eius insignibus proprietatibus", Leonhardi Euleri Opera Omnia, Ser. 1, Opera Mathematica, 6, 1921 (orig. date 1779), 350-369; Opera Mathematica, 15 (1927) [orig. date 1777], 268-297.

34. C. Truesdell. Ann. of Math. 46, 144-157 (1945).

35. S. Panda and B. K. Panda. Pramana 75, 393-402 (2010).

36. S. Ciccariello. J. Math. Phys. 45, 3353-3361 (2004).

37. P. Jodrá. Proc. R. Soc. A 464, 3081-3088 (2008).

38. L. Vepštas. Numer. Algorithms. 47, 211-252 (2008).

39. D. Cvijović. Physica A 389, 1594-1600 (2010).

40. J. M. Borwein, D. M. Bradley, D. J. Broadhurst, and P. Lisoněk. Trans. Amer. Math. Soc. 353, 907-941 (2001). 
